\title{
Self-reported dental pain and associated factors in Ugandan schoolchildren
}

\author{
S.N. Kiwanuka ${ }^{1,3}$ and A.N. Åstrøm ${ }^{1,2}$ \\ 1) Centre for international health, University of Bergen, Norway \\ 2) Department of Oral Sciences-Community Dentistry, University of Bergen, Norway \\ 3) Department of Dentistry, Faculty of Medicine, Makerere University, Uganda \\ Correspondence: Anne Nordrehaug Åstrøm, Center for international health, Armauer Hansen Building, N-5021 Bergen, Norway \\ Telephone: +4755974984 Telefax: +4755974979 e-mail: anne.nordrehaug@cih.uib.no
}

\begin{abstract}
There is a limited amount of research on the prevalence and determinants of subjective oral health indicators in children. Objective: to assess the prevalence of self-reported dental pain and to explore its relationship with socio-demographic characteristics in 10-14 year olds attending primary schools. Method: A cross-sectional survey was conducted during January-March 2004, including 11 public primary schools in Kampala, Uganda. A total of 614 children completed questionnaires administered in schools. Dental caries and plaque status were recorded in permanent teeth. Results: Experience with dental pain was confirmed by $42.1 \%$ boys and $52.3 \%$ girls. The crude prevalence of dental caries was $37.9 \%$ in boys and $42.1 \%$ in girls. Plaque was present on anterior teeth and $84.3 \%$ complained of at least one oral problem. Multiple logistic regression analysis revealed that reporting at least two oral problems $(\mathrm{OR}=2.7)$, being dissatisfied with dental appearance $(\mathrm{OR}=2.7)$ and having visited a dentist twice during the previous 3 years $(\mathrm{OR}=$ 2.2) were associated with higher odds of reported dental pain. Conclusion: A substantial proportion of school children had experience with dental pain. Dental pain associated positively with dental caries, subjective oral health indicators and dental attendance. Knowledge about the extent and significance of dental pain is important for the planning and evaluation of preventive and treatment efforts.
\end{abstract}

\section{INTRODUCTION}

Emerging consensus in the literature has identified three major dimensions of oral health related quality of life (OHRQoL); clinically assessed disease and impairments, disease and treatment specific symptoms and functional and psychological disability $(1,2)$. Over the years, several subjective oral health indicators have been developed for application in adults (3-6). There is a lack of OHRQoL measures designed for children, although paediatric oral disorders are numerous and likely to affect children's quality of life negatively $(7,8)$. Few attempts have been made to assess the prevalence and determinants of OHRQoL generally and dental pain particularly in the child populations of nonindustrialized countries. In this study, dental pain is considered to be synonymous with toothache and described as pain originating from innervated tissues within the tooth or immediately adjacent to it (9).

Untreated dental caries might lead to dental pain, which in turn results in impacts of affected play and sleep, avoidance of certain types of food and decreased school performance (9-11). In low-income countries like Uganda, the exposure to dental services is low, and toothache has been cited as a common reason for children to seek dental care $(12,13)$. Previous studies involving 13-19-yr-old Ugandan school children have provided evidence of high rates $(44 \%)$ of delayed treatment demand (dental visiting because of toothache), indicating a need for emergency care for later stages of dental caries (12). As a result of a growing consumption of foods and drinks with added sugars and inadequate oral health care services, it is expected that caries experience of children will increase in Uganda and other sub-Saharan African countries (14). Previous national estimates for Uganda have placed the mean DMFT (decayed, missed and filled teeth) for 12-year-olds at 0.5 in 1987, 0.4 in 1988 and 0.4 in 1993 (15). National averages mask differences within the country with mean DMFT estimates varying from 0.6 to 2.9 across urban and rural communities (16).

Most epidemiological studies of the dental health situation in child populations have inquired about dental pain by asking parents $(9,17)$. In the USA, $5 \%$ of 5 12-yr-olds reported some pain from their teeth/gums in the previous 3 months according to their parents (18). Among South Australian children aged 5-15 yr, $12 \%$ (5-yr-olds) and $32 \%$ (12-yr-olds) reported a history of toothache (19). Shepherd et al. (10) interviewed 8 -yr-old British children and found a prevalence of $47.5 \%$. In non-industrialized countries, the prevalence and severity of children's dental pain has usually been higher than the figures presented in UK, the USA and Europe. Ratnayake and Ekanayake (13) examined 8yr-old Sri Lanka children and found a lifetime prevalence of oral pain of $49 \%$ and $53 \%$ as reported by 
children themselves and their parents, respectively. Naidoo et al. (20) examined 8-10-yr-olds in the Western Cape of South Africa and found a prevalence of dental pain within the past two months as high as $70 \%$. In a recent study of 12-yr-old Ugandan school children from a rural sub-county, toothache in the last four weeks was reported by $36.5 \%$, whereas $20.2 \%$ and $6.4 \%$ needed a filling and one or more teeth extracted, respectively (15).

According to the biopsychosocial model dental pain is known to have both biological and psychosocial components. Thus, dental pain perceptions are complex functions of socio-demographic status, individual characteristics such as knowledge, beliefs and expectations, in addition to the principle pathological cause of dental caries $(5,21)$. Although found to be consistently associated with severity of tooth decay, conditions such as erosion, trauma and exfoliation of primary teeth can also give rise to dental pain. A review of the epidemiology of dental pain and dental caries in child populations has shown that dental pain is prevalent among children even in contemporary populations with historically low levels of caries experience (9). In the health and lifestyle survey conducted among Finnish adolescents, 1977-1997, no tendency for the prevalence of toothache to decline across time was recorded despite a corresponding decline in caries experience (22). Among 5-10-yr-old South Australian school children, experience of toothache at any time in a child's lifetime was reported by $9 \%$ of parents of subjects who had no clinical evidence of dental caries (19). In developed countries, toothache has been reported to be most prevalent in individuals of low income and education $(13,17,22)$. Moreover, the caries-toothache association is found to be strongest in populations with reduced access to dental care, in lower socioeconomic status groups and in populations where dental caries is largely untreated (9). Less frequent dental attendance patterns have been associated with low prevalence of reported dental pain among children in non-industrialized countries (13).

Description of the extent and distribution of dental pain is important when assessing the burden of dental diseases in children. Focusing on 10-14-year-old primary school children in Kampala, Uganda, this study aimed to assess the prevalence of dental pain and its association with dental caries experience, socio-demographic characteristics, oral hygiene, dental attendance and self-reported oral health. Socio-demographics as possible effect modifiers of the association between dental caries and dental pain was also investigated.

\section{MATERIAL AND METHODS}

\section{Study population and sampling method}

A cross-sectional study was conducted among children attending standard seven in primary schools in Kampala $(0.3 \mathrm{mg}$ fluoride/L), the capital city of Uganda.
The study was conducted during January-March 2004 using a structured questionnaire and a clinical examination. A list of all government (public) primary schools $(\mathrm{n}=13)$ within the Kampala central division (area $14.7 \mathrm{~km}^{2}$ ) was obtained from the Division Headquarters. Two primary schools with less than 30 children were excluded due to limited size leaving 11 schools to constitute the sampling frame of 2589 standard seven pupils. A sample size of 650 children was calculated based on an assumed prevalence of reported dental pain of $50 \%$, a standard error of $5 \%$ and a design effect of 2 (21). Lists of all students in standard 7 were obtained from the school authorities and every third student in each school was randomly selected to participate. This sampling strategy provided a sample that was self-weighting, implying that each participating student had the same probability of being selected into the study. Allowing for refusal to give informed consent and 701 signed letters were returned. The help of teachers was elicited in reminding the children to return the signed forms and to set an appropriate date for the data collection. A total of 67 pupils were excluded due to a wide age range (15-18 yr) and to being absent from school on the day of data collection. Twenty pupils who completed the questionnaire survey refused to be examined clinically and were also excluded from the study. The final participation rate for the main questionnaire survey and clinical study was $74 \%(n=614)$.

\section{Ethical considerations}

Ethical clearance was obtained from the ethical research committees in Norway and Uganda. Written permission to conduct the study was obtained from the Ministries of Health and Education in Uganda, local administration authorities and the school authorities. Written informed consent was obtained from the parent on behalf of their children.

\section{Clinical examination}

The clinical examination was carried out under field conditions in the class-room setting by one dentist (SNK), whereas a trained assistant recorded the observations. Students were examined whilst seated on a chair, using a head lamp as source of illumination. Initially visible plaque on anterior maxillary teeth was recorded. Dental probes and plane mouth mirrors were employed. The teeth were cleaned and dried with cotton roles before being examined for caries using the decayed, missing and filled tooth index (DMFT) as described by the World Health Organization (23). Caries was recorded as being present when a lesion in a pit/fissure or on a smooth surface had a detectable softened floor, undermined enamel, softened wall or a temporary filling in addition to sticky enamel lesions. A tooth was considered missing if there was a history of extraction due to pain and or the presence of a cavity. Lesions were recorded as present when a carious 
cavity was apparent on visual inspection under field conditions $(\mathrm{DMFT}=0$ and DMFT $>0$ ). Calibration exercise was carried out at the Institute for Pediatric Dentistry, Faculty of Dentistry, University of Bergen, Norway.

\section{Questionnaire survey}

The structured questionnaire comprised various sociodemographic and oral health related variables. The questionnaire was constructed and administered in English, which is the language of instruction in all formal academic institutions in Uganda. Health professionals reviewed the survey instrument for semantic, experiential and conceptual equivalence. Sensitivity to culture and selection of appropriate words were considered. The questionnaire was pilot tested and adjusted accordingly before being used in the field. The main researcher (SNK) and four trained assistants administered the questionnaire in schools as part of the classroom activity to provide a standard administration. Questions were read out loud one at a time while the participants filled in the responses on their own.

\section{Dependent variable}

Self-reported dental pain was assessed using one single question. The participants were asked whether or not they had experienced dental pain during the previous 12 months. Response categories were given as yes (1) and no (0).

\section{Independent variables}

Bleeding gums and sore mouth were inquired in terms of (1) yes and (0) no. A self-reported oral problem index was constructed from the two items. Aspects of the dwelling were assessed including fuel used for cooking as indicators of socioeconomic status. The predictor variables used in the analyses, their coding and the number of subjects (\%) according to categories are given in Table 1.

\section{Statistical analysis}

Data was entered using STAR OFFICE and transferred to SPSS version 13.0 for analyses. Univariate analyses were performed by use of chi-square statistics and logistic regression. Reproducibility was assessed using Cohen's kappa and Spearman's correlation coefficient. Multiple variable analysis was conducted using multiple logistic regression.

\section{RESULTS}

\section{Characteristics of participants}

A total of 614 students, $45.1 \%$ boys, mean age 12.4 , $\mathrm{SD}=1.0,59.6 \%$ younger $(10-12 \mathrm{yr})$ participated in the questionnaire survey and were examined for dental ries. Most of the younger students were girls. A majority confirmed brushing with toothpaste (98\%). In Uganda most of the commercialised toothpaste is fluoridated. Above three in five participants reported at least one oral symptom and 553 (90.1\%) were satisfied with their mouth and teeth. A majority, 346 (56.4\%) did not confirm dental attendance during the previous 3 years (Table 1$)$.

\section{Test-retest reliability}

Forty students $(50 \%$ boys, mean age $12.6, \mathrm{SD}=1.0)$ completed the questionnaire and were examined clinically a second time after one week. The examiner agreement for the clinical examination in terms of DMFT was found to be acceptable (Cohen's kappa = 0.75). Spearman's correlation coefficient across the questionnaire variables were 0.38 (toothache), 0.56 (satisfaction with oral condition), 0.53 (satisfaction with dental appearance), 0.84 (dental attendance), 0.74 (household energy source), and 1.00 (age and gender).

\section{Prevalence of caries experience, plaque and self- reported dental pain}

The first molars (270 teeth) were most frequently affected with untreated decay, closely followed by the second molars (220 teeth). The lower molars were more frequently affected than their upper counterparts (Figure 1). The mean DMFT was 0.98, SD = 1.6, range $0-15$. The prevalence of untreated dental caries, DT $>0$, was 235 (38.3\%), constituting $95 \%$ of the DMFT score. The age specific prevalence of caries experience (DMFT $>0)$, visible plaque and selfreported dental pain in boys and girls is depicted in Table 2. Dental pain was confirmed by 284 participants $(47.6 \%, 95 \%$ CI $43.7-51.5), 42.1 \%$ (95\% CI 36.3-47.9) boys and 52.3\% (95\% CI 48.0-58.0) girls. Among males, $47.9 \%$ versus $35.2 \%(p<0.05)$ of $10-12$ yr-olds and 13-14-yr-olds confirmed dental pain.

Table 1. Number (\%) of subjects by category on independent variables.

\begin{tabular}{llr}
\hline Variables & Categories (code) & N $(\%)$ \\
\hline Gender & Girl $(1)$ & $337(54.9)$ \\
Age & Boy $(2)$ & $277(45.1)$ \\
Household energy source & $10-12(1)$ & $366(59.6)$ \\
& $13-14(2)$ & $248(40.4)$ \\
& Electricity (0) & $159(26.0)$ \\
Dental visit last three years & Other (wood/ & $453(74.0)$ \\
& charcoal) $(1)$ & \\
Caries experience & Once $(2)$ & $346(56.4)$ \\
& Twice $(3)$ & $163(26.5)$ \\
Plaque score & DMFT $=0(0)$ & $105(17.1)$ \\
& $0<$ DMFT<3 $(1)$ & $159(59.8)$ \\
Dental appearance & DMFT $\geq 3(2)$ & $88(14.3)$ \\
Symptoms & No $(0)$ & $275(44.8)$ \\
& Yes $(1)$ & $339(55.2)$ \\
& Satisfied $(0)$ & $509(83.2)$ \\
& Dissatisfied $(1)$ & $103(16.8)$ \\
& None $(0)$ & $200(34.0)$ \\
& One $(1))$ & $281(47.8)$ \\
& Two $(2)$ & $107(18.2)$ \\
\hline
\end{tabular}




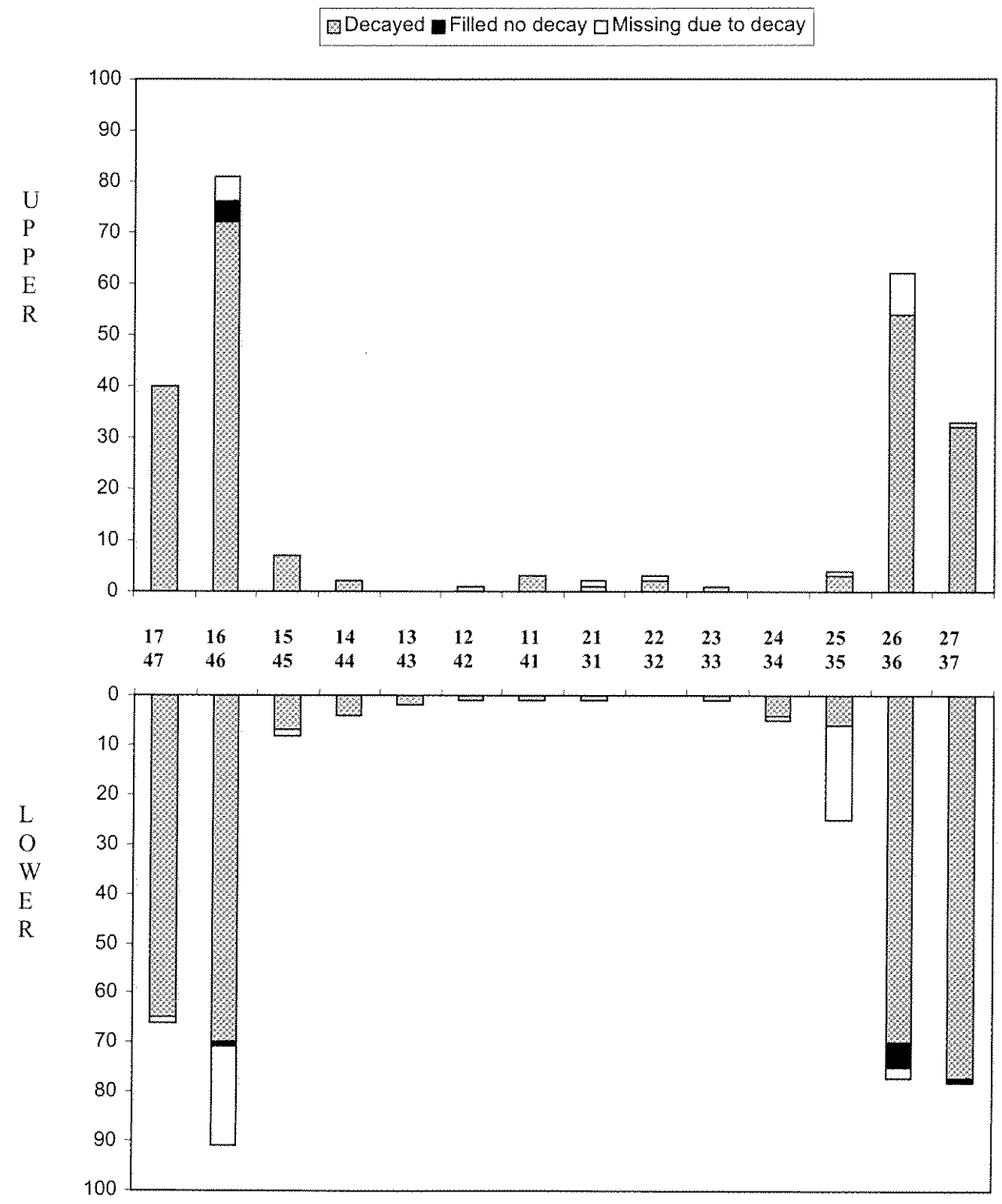

Figure 1. Number of decayed, missed and filled teeth according to tooth type in the upper and lower jaw.

Table 2. Prevalence of reported dental pain, prevalence of caries experience and prevalence of the presence of dental plaque by age and gender.

\begin{tabular}{lcccccc}
\hline & \multicolumn{3}{c}{ Boys } & \multicolumn{3}{c}{ Girls } \\
\cline { 2 - 7 } & All & $10-12$ & $13-14$ & All & $10-12$ & $13-14$ \\
\hline DMFT $>0$ & $37.9(105)$ & $33.1(49)$ & $43.4(56)^{*}$ & $42.1(142)$ & $36.7(80)$ & $52.1(62)^{*}$ \\
Plague present & $60.0(166)$ & $65.5(97)$ & $53.5(69)^{*}$ & $51.3(173)$ & $54.1(118)$ & $46.2(55)$ \\
Dental pain-yes & $42.1(112)$ & $47.9(69)$ & $35.2(43)^{*}$ & $52.0(172)$ & $52.3(112)$ & $51.3(60)$ \\
\hline
\end{tabular}

$* \mathrm{p}<0.05$

\section{Correlates of self-reported dental pain}

Caries free children reported experience with dental pain less frequently than their counterparts having DMFT $>0(42.0 \%$ versus $55.8 \%, \mathrm{p}<0.001)$. In children with dental pain experience, $45.4 \%$ had DT $>0$ and
$46.1 \%$ had never visited a dentist. Table 3 depicts the percentage of participants who reported dental pain by socioeconomic characteristics, oral health related behaviour and self-reported oral health in the total sample and separately for participants with and without dental caries experience. Boys tended to report 
Table 3. Percent (n) of participants who reported dental pain by socio-demographics, behavioural and clinical characteristics in the total sample and separately for subjects with and without dental caries experience.

\begin{tabular}{llll}
\hline & All \%(n) & DMFT $>0$ & DMFT $=0$ \\
\hline Age & & & \\
$10-12$ & $50.6(181)$ & $60.3(76)$ & $45.3(105)$ \\
$13-14$ & $43.6(103)$ & $50.9(58)$ & $36.0(45)^{*}$ \\
Gender & & & \\
$\quad$ Girls & $52.0(172)$ & $60.7(85)$ & $45.5(87)$ \\
$\quad$ Boys & $42.1(112)^{*}$ & $49.0(49)^{*}$ & $38.0(63)$ \\
Oral problems & & & \\
$\quad$ None & $37.5(75)$ & $47.6(39)$ & $30.5(36)$ \\
$\quad$ One & $47.6(131)$ & $53.3(57)$ & $44.0(74)$ \\
$\quad$ Two & $63.8(67)^{* *}$ & $72.1(31)^{*}$ & $58.1(36)^{* *}$ \\
Plaque & & & \\
$\quad$ no & $42.9(114)$ & $48.9(43)$ & $39.9(71)$ \\
$\quad$ yes & $51.4(170)^{*}$ & $59.9(91)$ & $44.1(79)$ \\
Dental appearance & & & \\
$\quad$ Satisfied & $42.9(212)$ & $50.8(99)$ & $37.8(113)$ \\
$\quad$ Dissatisfied & $70.3(71)^{* *}$ & $77.3(34)^{* *}$ & $649(37)^{* *}$ \\
Dental visits & & & \\
$\quad$ Never & $38.8(131)$ & $42.7(47)$ & $36.8(84)$ \\
$\quad$ Once & $57.0(90)$ & $69.9(51)$ & $45.9(39)$ \\
$\quad$ Twice or more & $62.4(63)^{* *}$ & $63.2(36)^{* *}$ & $61.4(27)^{*}$ \\
\hline **p $<0.001, * \mathrm{p}<0.05$ & & &
\end{tabular}

dental pain least frequently - at least those having experience with dental caries. Dental plaque, oral symptoms and children's global ratings of oral health were statistically significantly associated with dental pain. The prevalence of reported dental pain increased by increasing number of reported dental visits and more strongly among those having DMFT $>0$ than among their caries-free counterparts.

Table 4 depicts the unadjusted and adjusted odds ratios for having experienced dental pain according to clinical and non-clinical variables. Socio-demographics entered in the first step explained $1.8 \%$ of the variance in reported dental pain (Nagelkerke's $\mathrm{R}^{2}=$ 0.018 , Model Chi-Square 7.9, df 3, p = 0.047). Entering behavioural variables and self-reported oral health in a second step raised the explained variance to $14.5 \%$ $\left(\right.$ Nagelkerke's R ${ }^{2}=0.145$, Model Chi-Square $=66.3$, df $8, p=0.000$ ). Entering DMFT and plaque scores in the final step raised the explained variance by 1.6 percentage points (Nagelkerke's $\mathrm{R}^{2}=0.016$, Model Chi Square $=73.3, \mathrm{df}=10, \mathrm{p}=0.000$ ). In the final model, dental pain was found to be associated with reporting at least two oral problems $(\mathrm{OR}=2.7)$, being dissatisfied with dental appearance $(\mathrm{OR}=2.7)$ and having visited a dentist twice during the previous 3 years $(\mathrm{OR}=2.2)$. Frequency of dental visiting showed a dose-response relationship with reported pain with OR's of 1.9 and 2.2 for children confirming dental visits once and twice, respectively. A similar pattern was shown for the relationship between dental caries
Table 4. Dental pain regressed upon socio-demographic characteristics, subjective oral health ratings and dental status, unadjusted (unadj) and adjusted (adj) OR and 95\% CI.

\begin{tabular}{lcccc}
\hline & OR unadj & $95 \% \mathrm{CI}$ & OR adj & $95 \% \mathrm{CI}$ \\
\hline Age group & & & & \\
$10-12$ & 1 & & 1 & \\
$13-14$ & 0.7 & $0.5-1.0$ & 0.76 & $0.5-1.1$ \\
Gender & & & & \\
$\quad$ Girl & 1 & & 1 & \\
Boy & $\mathbf{0 . 7}$ & $\mathbf{0 . 4 - 0 . 9}$ & 0.6 & $0.5-1.1$ \\
Source of cooking & & & & \\
Electricity/gas & 1 & & 1 & \\
Other sources & 1.2 & $0.8-1.6$ & 1.1 & $0.6-1.6$ \\
Oral problems & & & & \\
None & 1 & & 1 & \\
One & $\mathbf{1 . 6}$ & $\mathbf{1 . 1}-\mathbf{2 . 1}$ & $\mathbf{1 . 5}$ & $\mathbf{1 . 1}-\mathbf{2 . 2}$ \\
Two & $\mathbf{2 . 9}$ & $\mathbf{1 . 8}-\mathbf{4 . 7}$ & $\mathbf{2 . 7}$ & $\mathbf{1 . 6}-\mathbf{4 . 9}$ \\
Dental appearance & & & & \\
Satisfied & 1 & & 1 & \\
Dissatisfied & $\mathbf{3 . 1}$ & $\mathbf{1 . 9}-\mathbf{4 . 9}$ & $\mathbf{2 . 7}$ & $\mathbf{1 . 5}-\mathbf{4 . 7}$ \\
Dental visits & & & & \\
Never & 1 & & 1 & \\
Once & $\mathbf{2 . 1}$ & $\mathbf{1 . 4 - 3 . 0}$ & $\mathbf{1 . 9}$ & $\mathbf{1 . 3}-\mathbf{2 . 8}$ \\
Twice & $\mathbf{2 . 6}$ & $\mathbf{1 . 6}-\mathbf{4 . 1}$ & $\mathbf{2 . 2}$ & $\mathbf{1 . 4}-\mathbf{3 . 5}$ \\
Caries & & & & \\
DMFT $=0$ & 1 & & 1 & \\
0 $<$ DMFT $<3$ & $\mathbf{1 . 7}$ & $\mathbf{1 . 1 - 2 . 3}$ & $\mathbf{1 . 4}$ & $\mathbf{1 . 0}-\mathbf{2 . 1}$ \\
DMFT $\geq 3$ & $\mathbf{1 . 9}$ & $\mathbf{1 . 2 - 3 . 1}$ & $\mathbf{1 . 8}$ & $\mathbf{1 . 1}-\mathbf{3 . 0}$ \\
Plaque & & & & \\
No & 1 & & 1 & \\
Yes & $\mathbf{1 . 4}$ & $\mathbf{1 . 1}-\mathbf{1 . 9}$ & $\mathbf{1 . 2}$ & $\mathbf{0 . 8}-\mathbf{1 . 7}$ \\
\hline
\end{tabular}

Nagelkerke's $R^{2}=15.5$

and reported pain with those having $0<\mathrm{DMFT}<3$ and $\mathrm{DMFT} \geq 3$ being 1.4 and 1.8 times more likely to report dental pain than their caries free counterparts. The interaction between source of fuel and DMFT status upon dental pain added significantly to the model $(\mathrm{p}=$ 0.012). Separate regression models for those having electricity (higher SES group) and those having wood/charcoal (lower SES group) as a source of fuel showed a statistically significant positive relationship between DMFT status and dental pain in the high SES group, only. Age seemed to modify the relationship between dental attendance and dental pain with a positive association being statistically significant among the 10-12-yr-olds only.

\section{DISCUSSION}

The results of the present study indicate that the prevalence of dental pain was high (47.6\%) among 10-14yr-old children attending primary school and varied systematically with attitudinal, behavioural and clinical characteristics of the study population. Compared to the European average DMFT score of 2.6 in 12-yrolds, the present mean DMFT score was low (24). It 
accords, however, with previously reported estimates of Ugandan children of comparable ages (16). Moreover, the very high proportion of unrestored teeth assessed (95\% of the DMFT score) is consistent with findings from other developing countries (25). It is not possible to assert that the present results demonstrate the crude impact of each explanatory variable considered since they could be biased by background confounding factors. However, the participants might be representative for the child population of 10-14-yr-olds in Kampala since about $90 \%$ of children of schoolgoing age (6-14 yr) attend primary schools according to national statistics (26). Because of the realities of life in Uganda, general population surveys of children and adolescents are difficult to conduct, and even the national oral health survey of 12 -year-olds was confined to school-going children (27).

Structured self-administered questionnaires as applied in this study have certain limitations (28). Bias due to social desirability, acquiescence and lack of recall are frequently encountered with children (7). Selfreported dental pain is subject to misclassification, because children fail to identify the pain as dental in origin and might include other conditions in their reports (9). Nevertheless, recently developed generic and disease specific oral health related quality of life questionnaires have demonstrated that with appropriate technique it is possible to obtain valid and reliable reports from children $(7,8)$. Age specific questionnaires have been recommended for 6-7, 8-10 and 1114-yr-olds since those groups are homogeneous in terms of roles and cognitive abilities. A study involving self-completion of dental self-report questionnaires by 6-9-yr-old children showed a high level of concordance between the child and caregiver and appeared to be clinically valid (29). Whereas the reliability coefficients observed in this study indicated moderate reproducibility across questionnaire variables, the positive association found between DMFT status and dental pain supports the validity of children's self-reports. To improve the validity of the single item dental pain measure utilized, further research should include additional questions about more recently experienced dental pain, its perceived causes and impacts.

Comparing the present prevalence rate across child populations should be done with caution due to the various time frames and age groups utilized in different studies. The prevalence estimated in this study accords with the lifetime prevalence (any toothache ever) reported among 5-15 year old Australian school children and that of 8-year-olds from the city of Harrow in England $(10,19)$. It was in accordance with pain experienced during the last four weeks among 8yr-olds from Sri Lanka (13). However, the prevalence of dental pain among 10-14-yr-old Ugandan school children was higher than that reported in their rural counterparts and also higher than the prevalence found in Brazilian school children of comparable age
$(17,15)$. Despite the various recall periods used, variation in disease patterns and severity might explain the difference observed among rural and urban Ugandan schoolchildren. Okullo et al. (30) observed a higher caries experience among slightly older adolescents in urban Kampala as compared to rural Lira and attributed this regional gradient to the availability of sugared snacks and drinks in towns (31).

Independent of the frequency of dental attendance, the results revealed a positive association between dental caries experience and reported dental pain. Obviously, children's dental pain could be avoided and family quality of life improved through strengthening of the preventive and therapeutic dental services for primary school children (32). The association was moderately strong, however, and only about half of the children with dental pain experience had untreated dental caries. Other possible causes of dental pain in this age group might have been trauma to teeth and eruption of permanent teeth or exfoliation of deciduous teeth. The present finding supports those presented in previous studies suggesting that caries experience is a consistent clinical correlate of dental pain in children $(9,13,17)$. Evidence suggest that low family income and educational level associate with increased dental pain in children after controlling for confounding factors (22). Incidentally, the lack of a social gradient as observed here might be unique to the participants investigated, confirming the social homogeneity of Kampala city children attending primary school. Children's socioeconomic status occurred as a significant modifier of the association between caries experience and reported dental pain with the strongest relationship found in children from higher socioeconomic status families. Previous studies of sub-Saharan African origin have identified a social gradient in dental caries experience with children from upper social classes being those most seriously affected (14). In contrast, contemporary evidence from industrialised countries have shown that the lower the material standard of living, the worse the oral health whatever measure are used to assess this, be they clinical or self-reported indicators (9). Moreover, it is noteworthy that children who had seen a dentist once or twice during the previous 3 years reported dental pain more often then their counterparts with no dental visits. Similar results have been reported previously and might be attributed to symptomatic dental attendance patterns and need for emergency care in later stages of dental caries rather than an unexpected response to dental treatment $(12,33)$.

In sum, the present study indicates that the prevalence of reported dental pain was high in 10-14-yr-old children attending primary school in Kampala. Although the average DMFT was low, untreated caries contributed $95 \%$ of children's caries experience. Dental pain was most frequent in children having untreated dental caries, being dissatisfied with their oral health and among those who had attended a dentist most 
frequently. The caries-dental pain relationship was stronger in children from higher socioeconomic backgrounds. Knowledge about the extent and significance of reported dental pain clearly indicates a need to strengthening preventive and therapeutic dental services among primary school children in Kampala.

\section{ACKNOWLEDGEMENTS}

The financial support from the Norwegian Research Council through Faculty of Dentistry, University of Bergen (Grant no 710004) is highly appreciated. We would like to thank the students who participated in the extensive data collection.

\section{REFERENCES}

1. Slade GD. The oral health impact profile. In: Slade GD, ed. Measuring oral health and quality of life. Chapel Hill: University of North Carolina, Dental Ecology, 1997.

2. Gift HC, Atchinson KA, Dayton CM. Conceptualising oral health and oral health related quality of life. Soc Sci Med 1997; 44: 601-608.

3. Cushing AM, Sheiham A, Maizels J. Developing socio-dental indicators - the social impact of dental disease. Community Dent Health 1986; 3: 3-17.

4. Atchinson KA, Dola TA. Development of the Geriatric Oral Health Assessment. Index J Dent Educ 1990; 54: 680-687.

5. Locker D, Miller Y. Subjectively reported oral health status in an adult population. Community Dent Oral Epidemiol 1994; 22: 425-430.

6. Adulyanon S, Vourapukjaru J, Sheiham A. Oral impacts affecting daily performances in a low dental Thai population. Community Dent Oral Epidemiol 1996; 24: 385-389.

7. Jokovic A, Locker D, Stephens M, Kenny D, Tompson B, Guyatt G. Validity and reliability of a questionnaire for measuring child oral health related quality of life. J Dent Res 2002; 81: 459-463.

8. Gherunpong S, Tsakos G, Sheiham A. Developing and evaluating an oral health related quality of life index for children; the CHILD-OIDP. Community Dent Health 2004; 21: 161-169.

9. Slade GD. Epidemiology of dental pain and dental caries among children and adolescents. Community Dent Health 2001; 18: 219-277.

10. Shepherd MA, Nadanovsky P, Sheiham A. The prevalence and impact of dental pain in 8-year-old schoolchildren in Harrow, England. Br Dent J 1999; 187: 38-41.

11. Levine RS, Pitts NB, Nugent ZJ. The fate of 1,587 un-restored carious deciduous teeth: a retrospective general dental practice based study from northern England. Br Dent J 2002; 193: 99-103.

12. Okullo I, Åstrøm AN, Haugejorden O. Social inequalities in oral health and in use of oral health care services among adolescents in Uganda. Int J Paed Dent 2004; 14: 326-335.

13. Ratnayake N, Ekanayake L. Prevalence and impact of oral pain in 8-year old children in Sri Lanka. Int J Paed Dent 2005; 15: 105-112.

14. Petersen PE. The World Oral Health Report 2003: continuous improvement of oral health in the $21^{\text {st }}$ century the approach of the WHO Global Oral Health programme. Community Dent Oral Epidemiol 2003; 31 (Suppl. 1): 3-24.

15. Nalweyiso N, Busingye J, Whitworth J, Robinson PG. Dental treatment needs of children in a rural subcounty of Uganda. Int J Paed Dent 2004; 14: 27-33.

16. Okullo I. Assessing oral impacts on daily performance, dental caries and aspects of oral care services among adolescents in Uganda. PhD thesis, University of Bergen, Creato Media, 2004.

17. Nomura LH, Bastos JLD, Peres MA. Dental pain prevalence and association with dental caries and socioeconomic status in schoolchildren, Southern Brazil, 2002. Braz Oral Res 2004; 18: 134-140.

18. Bailit HL. The prevalence of dental pain and anxiety; their relationship to quality of life. NY State Dent $J$ 1987; 53: 27-30.

19. Slade GD, Spencer AJ, Davies MJ, Burrow D. Intra-oral distribution and impact of caries experience among South Australian schoolchildren. Aust Dent J 1996; 41: 343-350.

20. Naidoo S, Chikte UME, Sheiham A. Prevalence and impact of dental pain in 8-10-year-olds in the western Cape. SADJ 2001; 56: 521-523.

21. Lwanga SK, Lemeshow S. Sample size determination in health studies. Geneva: World health Organization, 1991.

22. Honkala E, Honkala S, Rimpela A, Rimpela M. The trend and risk factors of perceived toothache among Finnish adolescents from 1977 to 1997. J Dent Res 2001; 80: 1823-1827.

23. World Health Organization: Oral health surveys, Basic Methods, $4^{\text {th }}$ edn. Geneva, 1997. 
24. WHO oral health country/area profile. Geneva: World Health Organization, http://www.whocollab.od.mah.se/ index.html.

25. David J, Wang NJ, Åstrøm AN, Kuriakose S. Dental caries and associated factors in 12-year old schoolchildren in Thiruvananthapuram, Kerala, India. Int J Paediatri Dent 2005; 15: 420-28.

26. Uganda Bureau of Statistics Education 2004. http://www.ubos.org/steducation.html.

27. Tirwomwe F, Ekoku Y, Manji F, Bælum V, Fejerskov O. Oral health in Uganda: Results from a National survey 1987. Kampala: Ministry of Health, Medical Research Institute, 1988.

28. Mc Dowell I, Newell C. Measuring health: A guide to rating scales and questionnaires, $1^{\text {st }}$ edn. New York: Oxford University Press, 1996.

29. Jamieson LM, Thomson WM, McGee R. An assessment of the validity and reliability of dental self-report items used in a national Child Nutrition Survey. Community Dent Oral Epidemiol 2004; 32: 49-54.

30. Okullo I, Åstrøm AN, Haugejorden O, Rwenyonyi C. Variation in caries experience and sugar intake among secondary school students in urban and rural Uganda. Acta Odontol Scand 2003; 61: 197-202.

31. Diehnelt DE, Kiyal HA. Socioeconomic factors that affect international caries levels. Community Dent Oral Epidemiol 2001; 29: 226-233.

32. Locker D, Jokovic A, Stephens M, Kenny D, Tompson B, Guyatt G. Family impact of child oral and orofacial conditions. Community Dent Oral Epidemiol 2002; 30: 438-448.

33. Vigild M, Petersen PE, Hadi R. Oral health behaviour of 12-year-old children in Kuwait. Int J Paed Dent 1999; 9: 23-29. 